\title{
Does the use of Robotic Technology Improve Surgeon Ergonomic Safety during TKA?
}

\author{
Laura Y. Scholl ${ }^{1}$, Emily Hampp ${ }^{1}$, Vincent Alipit ${ }^{1}$, Antonia F. Chen ${ }^{2}$, \\ Michael A. Mont ${ }^{3}$, and Anil Bhave ${ }^{4}$ \\ ${ }^{1}$ Stryker, Mahwah, NJ, USA \\ ${ }^{2}$ Birgham and Woman's Hospital, Boston, MA, USA \\ ${ }^{3}$ Lenox Hill Hospital, New York, NY, USA \\ ${ }^{4}$ Sinai Hospital Rubin Institute, Baltimore, MD, USA \\ laura.scholl@stryker.com, emily.hampp@stryker.com, vincent.alipit@stryker.com, \\ antoniachen1@gmail.com,mmont@northwell.edu, anilbhave@yahoo.com
}

\begin{abstract}
Surgeon physical stress in the operating room is a known potential cause of musculoskeletal overuse injuries, specifically in surgeons who perform total knee arthroplasty (TKA). Injuries have been attributed to ergonomically challenging postures. This study compared surgeon lower back and shoulder posture between manual TKA (MTKA) and robotic assisted TKA (RATKA).

Two surgeons performed a total six MTKA and six RATKA on a set of cadaveric knees. Movement and EMG sensors were secured to each surgeon to monitor lower back and shoulder movements, as well as muscle activities. Data was analyzed and activities were assessed as low, medium, or high risk, providing a score between 0-lowest and 16-highest. Risk data was compared between MTKA and RATKA for three separate surgical tasks: 1-bone cut preparation $\&$ cutting $($ MTKA = placement of cutting jigs, bone cutting, RATKA = array placement, bone registration, bone cutting), 2-knee balancing and 3-trialing.

Overall, there were more high-risk shoulder than lower back activities in MTKA and RATKA. More high-risk movement and EMG stimulation were measured in the dominant shoulder than the non-dominant. When lower back and shoulder data were combined, highest risk task was bone cut preparation \& cutting (MTKA: 13 vs. 6 vs. 6 and RATKA: 11 vs. 8 vs. 6), with a higher risk for MTKA than RATKA.

Poor posture can be a potential cause for surgeon work-related injuries. This study evaluated which tasks presented highest risk to surgeon ergonomic safety while performing TKA, and found lower overall ergonomics risk for performing RATKA vs. MTKA. Although this study provides data indicating reduced ergonomic risk with RATKA, additional studies in the operating room need to be performed.
\end{abstract}


Does the use of Robotic Technology Improve Surgeon Ergonomic Safety during TKA? L. Scholl et al.

\section{Introduction}

Surgeon physical stress in the operating room is a known potential cause of musculoskeletal overuse injuries [1]. These injuries can be exacerbated, specifically in surgeons who perform very high activity operations that require substantial physical stamina, such as total knee arthroplasty (TKA). Injuries have been attributed to ergonomically challenging postures, which can consequently affect a surgeon's operative career [2]. Thus, there is a need to explore ergonomic health and workload demands when performing TKAs. With the development of robotic assisted TKA, it is also important to understand the influence of surgical methods on posture. This study compared surgeon lower back and shoulder posture between manual TKA (MTKA) and robotic assisted TKA (RATKA).

\section{Materials and Methods}

Twelve TKAs (6 cadaver specimens) were performed by two fellowship-trained surgeons who were right hand dominant. For each cadaver, MTKA was performed on one knee and RATKA on the contralateral knee. Surgeons alternated between MTKA and RATKA, until three of each were completed. Sets of wearable movement and electromyography (EMG) sensors were secured to each surgeon to monitor lower back and shoulder movements, as well as muscle activities. For lower back assessments, 2 EMG sensors were placed on the erector spinae muscles, 1 movement sensor was placed on the T12/L1 region, and 1 movement sensor was placed on the L5/S1 region. For shoulder assessments, 2 EMG sensors were placed on both trapezius muscles and 2 movement sensors were placed on the antero-lateral surfaces of both humeri.

The movement sensors tracked range of movement, repetitions, and sustained positions for flexion, extension, and lateral flexion of the lower back and elevation of the shoulders. The EMG sensors assessed standard voluntary muscle contractions. The data was analyzed, and activities were assessed as low, medium, or high risk based on standards listed in the Australian manual handling code of practice [3]. The posture and EMG risk data were combined and scored between 0 (lowest) and 16 (highest). Risk data was compared between MTKA and RATKA for three separate surgical tasks: (1) bone cut preparation \& cutting, (2) knee balancing and (3) trialing. Where bone cut preparation \& cutting consists of placement of cutting jigs and bone cutting for MTKA whereas for RAKTA it consists of array placement, bone registration and bone cutting.

\section{Results}

Overall, there were more high-risk shoulder than lower back activities in both MTKA and RATKA. Specifically, EMG values for the lower back were consistently within the low-risk zone, whereas, they reached the high-risk zone for the shoulders during bone cut preparation, cutting, and trialing. More high-risk movement and EMG stimulation were measured in the dominant shoulder than the nondominant shoulder.

When lower back and shoulder data were combined, the highest risk task was bone cut preparation $\&$ cutting compared to knee balancing and trialing (MTKA: 13 vs. 6 vs. 6 and RATKA: 11 vs. 8 vs. 6 ), and bone cut preparation \& cutting was higher risk for MTKA than RATKA (13 vs. 11). When further evaluated, it was noted that dominant shoulder ergonomics had increased risk compared to the nondominant shoulder and lower back, which was true between MTKA and RATKA. Additionally, it was noticed that this difference in dominant shoulder ergonomics mostly occurred during the femoral bone cutting process. RATKA had reduced overall risk during femoral bone cutting (13 vs. 9), which was 
Does the use of Robotic Technology Improve Surgeon Ergonomic Safety during TKA? L. Scholl et al.

attributed to reduced percentage of time in high-risk range of motion (ROM) (30 vs. 21\%) and repetitions per minute in high-risk ROM (2.1 vs. 0.4).

\section{Discussion and Conclusion}

It is well-documented that poor posture can be a potential cause for surgeon work-related injuries [4]. Outside of orthopaedics, the use of robotics laparoscopic surgery has been shown to encourage postures that are more ergonomic, reducing a surgeon's risk for musculoskeletal injury [5]. This study evaluated which tasks presented highest risk to surgeon ergonomic safety while performing TKA and found lower overall ergonomics risk for performing RATKA vs. MTKA. The highest ergonomic risks occurred during bone cut preparation and cutting. This increased risk was attributed mostly to ergonomics of the dominant shoulder. Reduced risk with RATKA may be associated with how the surgeon's arm is oriented when performing femoral bone cuts. The robotic assisted allows the surgeon to reduce their range of movement and repetitive movements. Although this study provides data indicating reduced ergonomic risk with RATKA, additional studies in the operating room tracking movements and muscle activities of the primary surgeon and first assist need to be performed.

\section{References}

[1] Alqahtani SM, Alzahrani MM, Tanzer M. Adult reconstructive surgery: a high-risk profession for work-related injuries. J Arthroplasty. 2016;31:1194-1198.

[2] Davis WT, Sathiyakumar V, Jahangir AA, Obremskey WT, Sethi MK. Occupational injury among orthopaedic surgeons. J Bone Joint Surg Am. 2013;95:e107(1-6).

[3] Code of Practice for Manual Handling. No. 25, 20 April 2000. Occupational Health and Safety Act 1985.

[4] Davis WT, Sathiyakumar V, Jahangir AA, Obremskey WT, Sethi MK. Occupational injury among orthopaedic surgeons. J Bone Joint Surg Am. 2013;95:e107(1-6).

[5] Yu D, Dural C, Morrow MM, Yang L, Collins JW, Hallbeck S, Kjellman M, Forsman M. Intraoperative workload in robotic surgery assessed by wearable motion tracking sensors and questionnaires. Surg Endosc. 2017 Feb;31(2):877-886. 